IEEE Instrumentation and Measurement

Technology Conference

Anchorage, AK, USA, 21-23 May 2002

\title{
Texture Characterization of the Visual Appearance of Paintings
}

\author{
Yang Cai<ycai@cmu.edu> and Mel Siegel<mws@cmu.edu> \\ Human-Computer Interaction Institute and Robotics Institute
}

Carnegie Mellon University

\begin{abstract}
A texture characterization method has been explored to model the visual appearance of paintings, before and after a surface treatment. A preliminary experiment has been conducted to test the texture feature vectors before and after varnishing with Grumbacher's Picture Varnish liquid. Numerical functions, such as level of harmony (energy) and level of disorder (entropy) are computed. The results show that both functions are sensitive to the effect of varnishing, where energy function is the most sensitive measurement, and entropy function shows that varnishing increases painting's contrast. The findings are consistent with the art conservationists' experience.
\end{abstract}

\section{Introduction}

People take works of art for granted, assuming that they will last forever in museums. But in reality, they can have short lives. Preserving artwork by identifying the art materials' qualities and the environmental conditions that cause artwork to degrade is a major field of research in art conservation.

Dynamic color measurement for paintings has been extensively studied. For example, the micro-light-fastness tester [Whitemore, 1996] measures the light-fastness of individual pinpoint of light measuring a fraction of a millimeter onto a tiny spot on the painting. It can determine how quickly a specific color will fade. The VASARI project [Saunders, 1993] uses digital camera and color separation method to determine the color coordinates. However, there are more intrinsic qualities other than color. We have found that the

change of material texture is also an important quality that is caused by cracks or overlay varnishing, etc. Computational pattern recognition method has been developed to classify the cracks on the painting. [Bucklow, 1999] The method uses the heuristic and algorithmic representations, based upon the Repertory Grid and Bezier curves respectively. In addition the specific crack classification study, many researches have been done in optical surface roughness measurement. Most of them are based on Bidirectional Reflectance Distribution
Function (BRDF) and the Bidirectional Texture Function (BTF) which models the visual appearance with four parameters: two each for viewing angle, illumination directions. [Dana, 2000, Kaya, et al 1995] The combination of viewing and illumination angles is tremendous for the experiment design. In addition, these two methods assume that the measured texture is fairly homogenous. In this paper, we present a simplified method that uses the fixed viewing and illumination angles and a vector of texture feature measurements, including Entropy (disorderness) and Energy (homogenity). The advantages of these methods are that it's simple and it yields objective and multidimensional textural characterization that eventually might go beyond the capacity of human perceptions. For example, monitoring art works in real-time, or treatment evaluation at a large scale, etc.

\section{Process}

A preliminary experiment has been conducted to test the numerical expressions of the texture features before and after varnishing with Grumbacher's Picture Varnish liquid. One of the first author's oil paintings was used as a test bed (Figire1). The painting was covered by a white paper where seven sampling windows were cut. (Figure 2) The camera was $640 \times 480$ CCD camera with USB connection to a laptop. The light source was a 4-head LED touch light. To simplify the experiment, only one lighting angle (45 degree) and one camera viewing angle (90 degree) was used during the experiment.

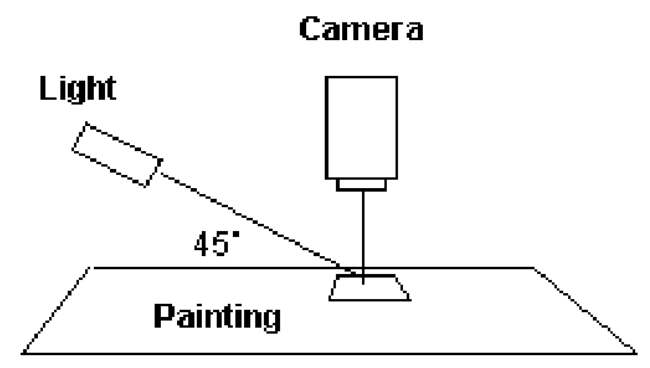

Figure 1. System Setup 


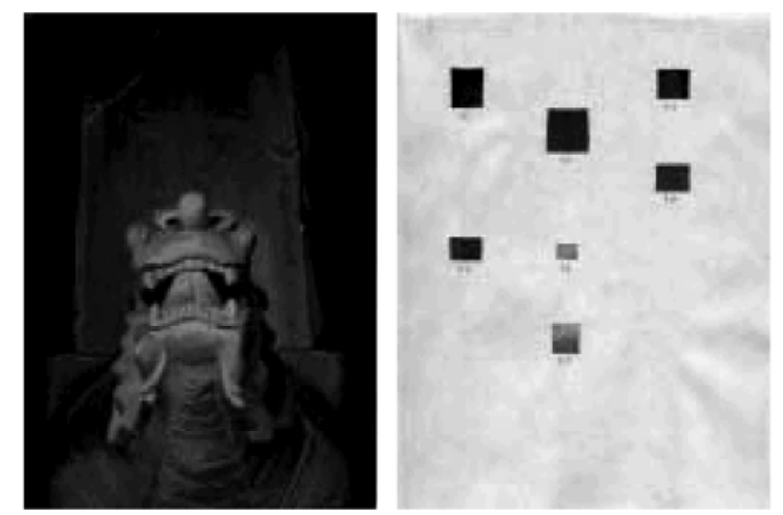

Figure 2. The Painting (left) and Sampling Windows (Right)

Before varnishing, seven sample images were captured and saved at the computer. Then the oil painting was varnished with a brush three times and waited until the varnishing coat dried. Then the painting was covered by the paper mask again and seven sample images were collected.

In this study, a texture feature space is composed with a set of texture feature descriptions in form of vectors.

$$
\mathbf{H}=\left[\mathrm{H}_{1}, \mathrm{H}_{2}, \mathrm{H}_{3}, \ldots, \mathrm{H}_{\mathrm{n}}\right]
$$

For example, the texture features include randomness, coarseness, linearity, periodicity, contrast, harmony, primitive length, etc. At this stage, we have implemented two of them: the level harmony (Energy) and level of disorder (Entropy).

$$
\begin{gathered}
\mathrm{H}_{\text {entropy }}=\underset{\mathrm{i} \mathrm{j}}{\sum \sum \mathrm{M}[\mathrm{i}, \mathrm{j}] \log (\mathrm{M}[\mathrm{i}, \mathrm{j}])} \\
\mathrm{H}_{\text {energy }}=\underset{\mathrm{i} \mathrm{j}}{\sum \sum[\mathrm{i}, \mathrm{j}] /(1+|\mathrm{i}-\mathrm{j}|)}
\end{gathered}
$$

where, $\mathrm{M}$ is a gray level co-occurrence matrix (GLCM) that contains information about the position of pixels having similar gray level values. The idea is to scan the image and keep track of how often pixels that differ by $\Delta z$ in value are separated by a fixed distance $d$ in position. [Parker, 1997] A MATLAB program was used to implement the algorithm.

\section{Results}

The preliminary results contain data analysis from seven sample image pairs, including those are taken before and after the varnishing.

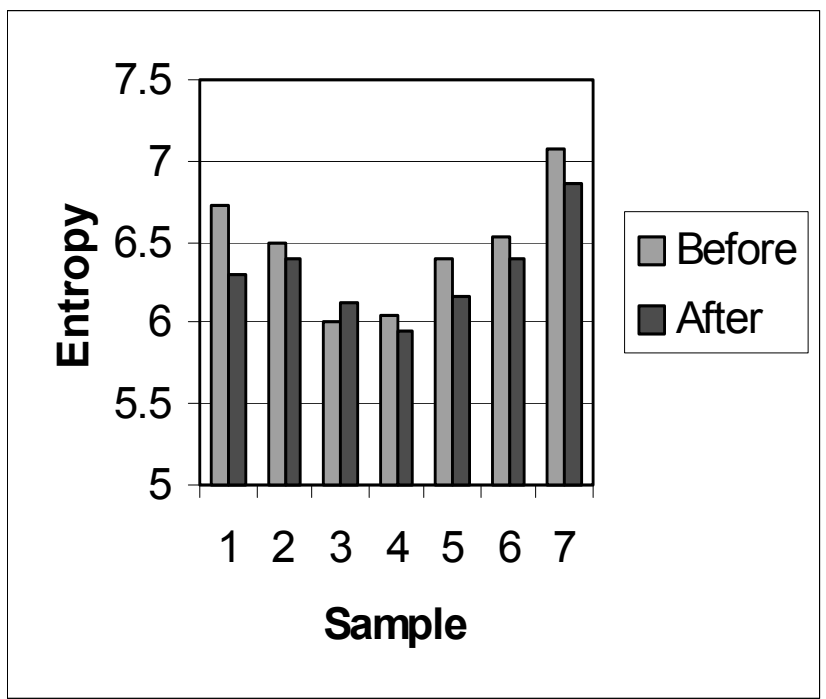

Figure 3. Entropy (Disorder)

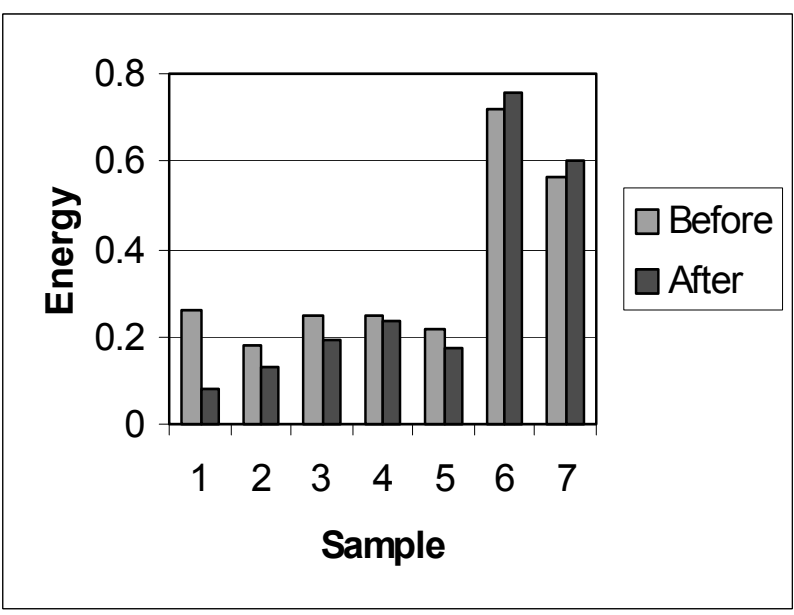

Figure 4. Energy (Harmony)

Table 1 Entropy Data

\begin{tabular}{|c|c|c|}
\hline Sample & Before & After \\
\hline 1 & 6.7155 & 6.298 \\
\hline 2 & 6.4982 & 6.4007 \\
\hline 3 & 6.0046 & 6.1154 \\
\hline 4 & 6.0547 & 5.9531 \\
\hline 5 & 6.3972 & 6.1604 \\
\hline 6 & 6.5354 & 6.3981 \\
\hline 7 & 7.0825 & 6.8573 \\
\hline
\end{tabular}


Table 2 Energy (Harmony) Data

\begin{tabular}{|c|c|c|}
\hline $\begin{array}{c}\text { Sampl } \\
\mathbf{e}\end{array}$ & Before & After \\
\hline 1 & 0.2607 & 0.082 \\
\hline 2 & 0.1822 & 0.1303 \\
\hline 3 & 0.2465 & 0.1933 \\
\hline 4 & 0.247 & 0.2369 \\
\hline 5 & 0.2192 & 0.1762 \\
\hline 6 & 0.7192 & 0.7572 \\
\hline 7 & 0.5652 & 0.6031 \\
\hline
\end{tabular}

First, it is found that both functions are sensitive to the effect of varnishing, where Energy function is the most sensitive measurement. Relatively it needs less data and provides more intuitive numerical results.

Second, the Entropy function shows that the values after varnishing are smaller, which indicates that varnishing makes a painting texture more clear. Also, data from the Entropy function appears to be more consistent than Energy function.

Third, from Energy function, it is found that the effect of varnishing is not uniform. In dark areas, varnishing makes the area darker, vice versa, which is consistent to the experience of art conservationists' observations.

Finally, the major problem of this measurement is that the variation of the values from both functions are not consistent cross the painting because the measurement interacts with the content of the painting, such as dark or light areas, boundaries, etc. How to calibrate those functions is a challenge. The potential solutions would be using better texture feature description functions, or better combinations of the description functions.

\section{Discussions}

The statistical texture description functions are useful to provide objective surface textural characterization measurement. However, art conservationists or material surface scientists use their own terminology, such as glossiness, "orange pill", etc. Therefore, it is desirable to convert the digital description data to the terms that experts are familiar with. On the other hand, it is also an opportunity to expend the scope of texture feature descriptions to new areas where there is no match in any conventional ways, such as fractal texture description, etc.

The gray scale co-occurrence matrix (GLCM) describes second order image statistics and works well for a large variety of textures for a survey of texture feature description. Good properties of the method are the description of spatial relations between tonal pixels, and invariance to monotonic gray-level transformations. On the other hand, it does not consider primitive shapes, and therefore cannot be applied to the texture with large primitives. Small sampling windows are necessary. Memory requirements are another weakness. But for the off-line texture characterization measurement, it is still acceptable in terms of computing resources.

\section{References}

Amots, H., (1994) Machine Vision Monitoring of Plant Nutrition, Ph.D. Dissertation, UMI, Order Number 9501704

Bucklow, S., (1999), The Description and Classification of Craquelure, Studies in Conservation 44 (1999), 233-244

Dana, K., and Ginneken, B., (1997), Reflectance and Texture of Real-World Surfaces, Proceedings of IEEE, CVPR'97

Elias, M. and Menu, M., (2000), Experimental Characterization of a Random Metallic Rough Surface by Spectrophotometric Measurements in the Visible Range, Optics Communications, 180 (2000) 191-198

Naya, S.K. and Oren, M., (1995), Visual Appearance of Matte Surfaces, Science, Vol. 267, pp. 1152-1156, Feb. 1995

Parker, J.R., (1997) Algorithms for Image Processing and Computer Vision, Wiley Computer Publishing

Saunders, D. and Cupitt, J., (1993) Image Processing at the National Gallery: The VASARI Project, National Gallery Tech Bull., 14, 7285,1993

Sonka, M. et al, (1999) second edition, Image Processing, Analysis, and Machine Vision, PWS Publishing

Whitemore, P, (1996), Ticking Time Bombs in the Art World, www.cmu.edu/cmri/spr_96Highlights.html 
Appendix A: Text samples - samples on the first row are unvarnished; samples below are varnished.

1
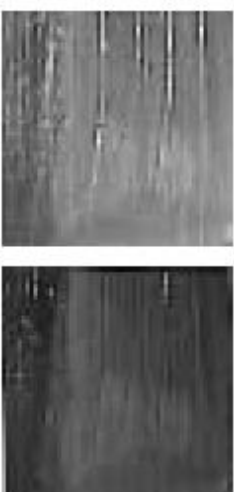

1
2
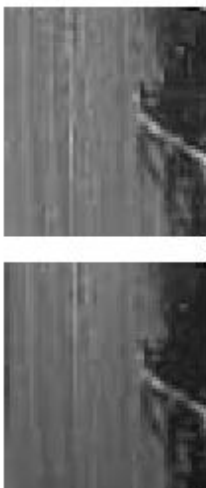

2
3
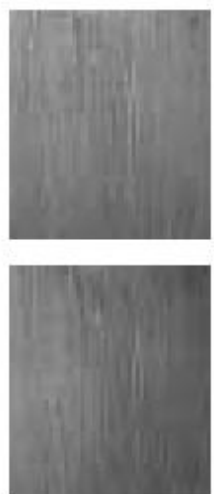

3
4
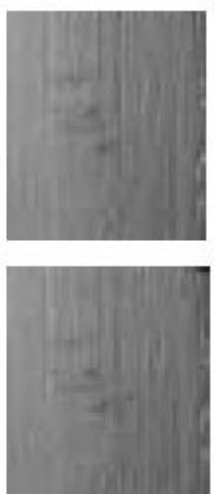

4
5
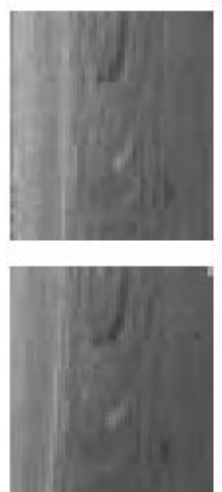

5
6

7
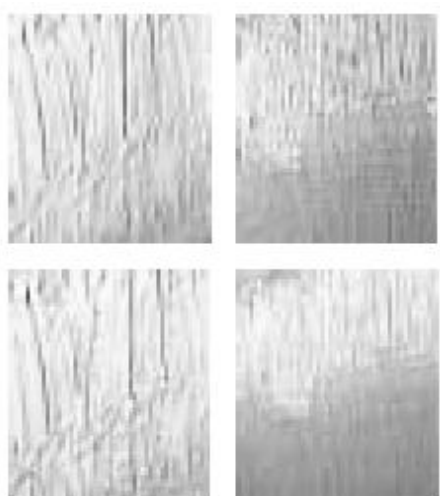

6

7 